\title{
METHOD FOR EVALUATION OF OUTAGE PROBABILITY ON RANDOM ACCESS CHANNEL IN MOBILE COMMUNICATION SYSTEMS
}

\author{
Martin Kollár *
}

\begin{abstract}
In order to access the cell in all mobile communication technologies a so called random-access procedure is used. For example in GSM this is represented by sending the CHANNEL REQUEST message from Mobile Station (MS) to Base Transceiver Station (BTS) which is consequently forwarded as an CHANNEL REQUIRED message to the Base Station Controller (BSC). If the BTS decodes some noise on the Random Access Channel (RACH) as random access by mistake (so- called 'phantom RACH') then it is a question of pure coincidence which 'establishment cause' the BTS thinks to have recognized. A typical invalid channel access request or phantom RACH is characterized by an IMMEDIATE ASSIGNMENT procedure (assignment of an SDCCH or TCH) which is not followed by sending an ESTABLISH INDICATION from MS to BTS. In this paper a mathematical model for evaluation of the Power RACH Busy Threshold (RACHBT) in order to guaranty in advance determined outage probability on RACH is described and discussed as well. It focuses on Global System for Mobile Communications (GSM) however the obtained results can be generalized on remaining mobile technologies (ie WCDMA and LTE).
\end{abstract}

K e y w ords: phantom RACH, GSM, Rayleigh-distribution, outage probability

\section{INTRODUCTION}

In order to access the cell in all mobile communication technologies a random-access procedure is used. For example in GSM this is represented by sending the CHANNEL REQUEST message (request for a Stand Alone Dedicated Control Channel (SDCCH) or Traffic Channel $(\mathrm{TCH})$ ) via the Random Access Channel (RACH) from mobile station (MS) to BTS which is consequently forwarded as an CHANNEL REQUIRED message to the BSC. The CHANNEL REQUIRED message contains the so-called random access information from the CHANNEL REQUEST which consists of the 'establishment cause' and a random bit sequence. If eg the BTS wrongly decodes this message (eg determines the wrong establishment cause due to a bad radio interface) it forwards the wrong information to the BSC. Moreover, if the BTS decodes some noise on the RACH as random access by mistake (the so-called 'phantom RACH') then it is a question of pure coincidence which 'establishment cause' the BTS thinks to have recognized. A typical phantom RACH is characterized by an IMMEDIATE ASSIGNMENT procedure (assignment of an SDCCH or TCH) which is not followed by an ESTABLISH INDICATION (is sent only if the SDCCH or TCH was successfully seized by the MS) $[1,2]$.

A prior art method of detecting phantom RACHs, known from networks implementing GSM, uses a twostaged procedure, which is based on determinations of two parameters: 1 . The parameter RACHBT, which defines a threshold for the received signal level during network access. A signal level exceeding this threshold is interpreted as a busy RACH. 2. Signal-to-noise ratio (SNR), where indications for channel access requests whose SNR does not exceed the threshold are filtered out. The SNR, which is measured indirectly via BER of the synchronization sequence bits within the RCH burst, plays a dominant role in detection of phantom RACHs. In GSM the number of synchronization sequence bits is equal to 41 as it can be seen also in Fig. 1.

Despite there are some other methods improving the detection of the phantom RACHs as described in $[1,2]$ the mathematical model describing this phenomenon and related scientific papers from that area are still missing.

\section{MATHEMATICAL MODEL FOR PHANTOM RACH}

As mentioned in the previous chapter, phantom RACH represents case when the BTS decodes some noise on the $\mathrm{RACH}$ as random access. The existence of the noise can be caused when more than one MS (Mobile Station) sends the CHANNEL REQUIRED message at the same time or by interference from neighbouring cells and the influence of white noise will be neglected. Let us consider that $r(t)$ represents the above mentioned noise in time domain according to the following formula

$$
r(t)=\sum_{n=1}^{N} c_{n} \cos \left(2 \pi f t+\varphi_{n}\right)
$$

\footnotetext{
* CMT OP NW TAR, Siemens Program and System Engineering, Ltd. Trieda SNP 37, 04100 Košice, Slovakia, martin.kollar@nsn.com
} 


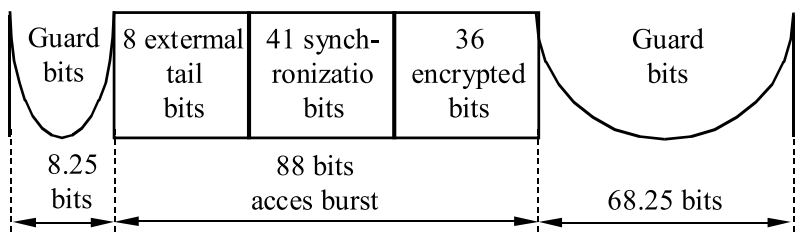

Fig. 1. The RACH Burst (duration $312.2 \mu \mathrm{s}$ ( 88 bits))

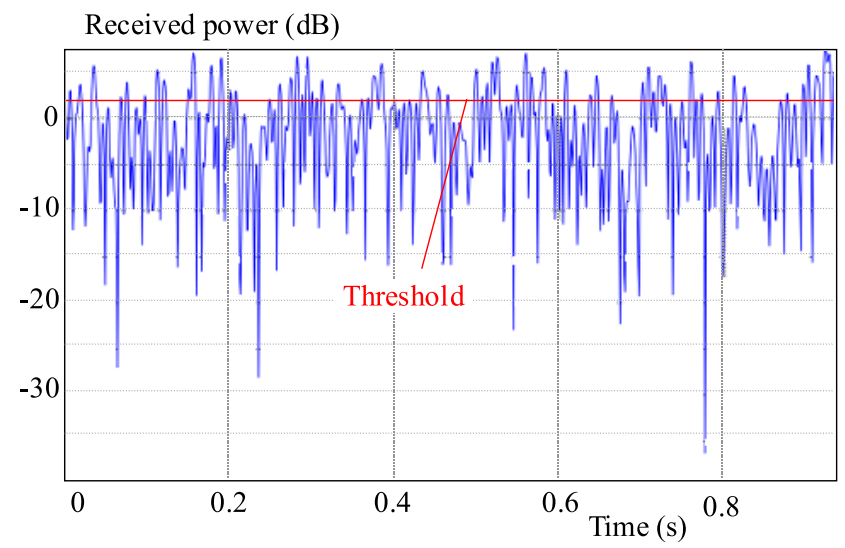

Fig. 2. Received power of $|r(t)|$ as function of time (an example)

where $N$ represents number of components which contribute for resultant noise, $c_{n}$ is the amplitude of the $n$-th component and $\varphi_{n}$ its phase, $f$ is the frequency of radio signal and $t$ represents time.

An in-phase-quadrature representation of the form

$$
r(t)=I(t) \cos (2 \pi f t)-Q(t) \sin (2 \pi f t),
$$

can be found with in-phase component

$$
I(t)=\sum_{n=1}^{N} c_{n} \cos \left(\varphi_{n}\right)
$$

and quadrature phase component

$$
Q(t)=\sum_{n=1}^{N} c_{n} \sin \left(\varphi_{n}\right) .
$$

Thus both the inphase and quadrature component, $I(t)$ and $Q(t)$ can be interpreted as the sum of many (independent) small contributions. For sufficiently many reflections (large $N$ ), the Central Limit Theorem [3] now says that the inphase and quadrature components tend to a normal distribution of their amplitude. $I(t)$ and $Q(t)$ appear to be independent and identically distributed.

As given in [4], absolute value of a random variable consisting of two orthogonal components normally and independently distributed will be Rayleigh-distributed. Therefore the probability density of the absolute value $\rho\left(\sqrt{I^{2}+Q^{2}}\right)$ of $r(t)$ is described by the Rayleigh function as follows

$$
g(\rho)=\frac{\rho}{\sigma^{2}} e^{-\frac{\rho^{2}}{2 \sigma^{2}}},
$$

where $\sigma$ represents so called root mean square (RMS) of the noise $r(t)$ (mathematically expressed as $|r(t)|$ ). The corresponding power of the $|r(t)|$ is then given as $\sigma^{2}\left(\mathrm{RMS}^{2}\right)$.

An example of the received power of $|r(t)|$ is shown in Fig. 2, where all cases when the received power exceeds given threshold can lead to outage ${ }^{1}$ on $\mathrm{RACH}$ (phantom $\mathrm{RACH})$.

Now we will consider that the threshold $Z$ is expressed in the same physical unit as the signal $r(t)$. Then for the outage probability on $\mathrm{RACH}$ it follows that

$$
p(\rho>Z)=\int_{Z}^{\infty} g(\rho) \mathrm{d} \rho .
$$

It means that outage probability is equal to the probability that amplitude of $r(t)$ exceeds the threshold $Z$. From (5) and (6) we have that

$$
p(\rho>Z)=e^{-\left(Z^{2} / 2 \sigma^{2}\right)}
$$

or considering that $P_{T H}=Z^{2}$ is the threshold in Watts. The threshold RACHBT is given in $\mathrm{dBm}$. To use this parameter in (8) the following formula must be applied $P_{T H}=10^{(R A C H B T / 10-3)}$

$$
p\left(\rho>P_{T H}\right)=e^{-\left(P_{T H} / 2 \sigma^{2}\right)} .
$$

The outage probability on RACH can be very easily measured in BSS using already existing measurement counters and is given by the following formula

$$
p(\rho>Z)=\frac{586 \times 10^{-6}\left(N_{C H R}-N_{E I}\right)}{0.03(T / 0.24)}
$$

where $N_{C H R}$ represents the number of CHANNEL REQUIRED messages, which is the message the BTS sends to the BSC when the BTS has received the CHANNEL RUEQEST message (request for an SDCCH or TCH) via the RACH, decreased about the cases when a valid CHANNEL REQUIRED messages could not be followed by an IMMEDIATE ASSIGNMENT procedure because of SDCCH, TCH or AGCH congestion. The $N_{E I}$ is the number of ESTABLISH INDICATION messages (is sent only if the SDCCH or TCH was successfully seized by the MS). The $T$ represents the observation period, $586 \mu \mathrm{s}$ is duration of one RACH burst including also guard intervals (see Fig. 1), $0.24 \mathrm{~s}$ represents duration of one TDMA frame and $0.03 \mathrm{~s}$ represents duration of $\mathrm{RACH}$ burst within one TDMA frame ${ }^{2}$.

\footnotetext{
${ }^{1}$ The outage probability means a probability that a noise at the BTS RF input will be evaluated as a valid input access request

${ }^{2}$ Equation (9) is referred to a standard GSM configuration when one slot with a standard duration $30 \mathrm{~ms}$ from a TDMA frame (lasting $240 \mathrm{~ms}$ ) is reserved for RACH
} 


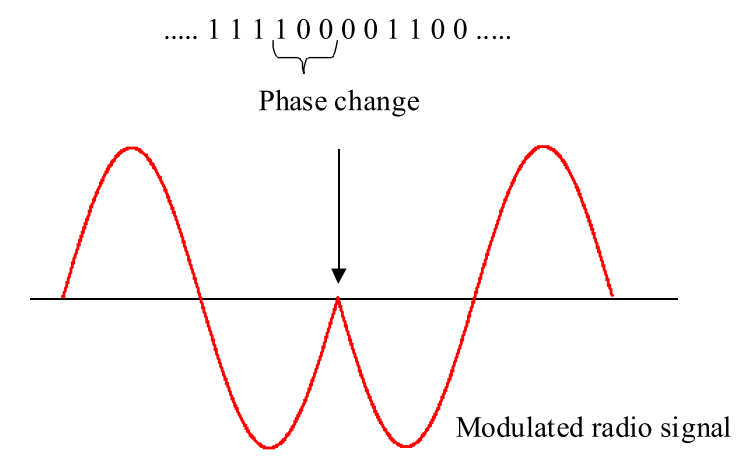

Fig. 3. The principle of 8 PSK modulation (one symbol includes 3 bits)

From (8) it follows that the $\sigma^{2}$ can be determined from the known outage probability and threshold $P_{T H}$. On the other hand with the knowledge of this power the threshold $P_{T H}$ may be calculated for in advance determined outage probability on RACH. There is only one restriction - the threshold $P_{T H}$ can not exceed the RXLEVAMI, which is the minimum received downlink level at the MS required for access to the network. The RXLEVAMI is sent by BTS to MS.

It should be noted that existing counter in BSS will not measure all random accesses on $\mathrm{RACH}$ caused by the noise because there is a limitation when the access signal is equalized and a training sequence of bits in the equalized access signal is compared to a reference sequence of bits. A burst confidence metric is obtained in the comparison by summing the number of matching bits. The RACH burst is discarded if the burst confidence metric is less than a threshold number ${ }^{3}$. Let us consider that $\sigma_{\text {Total }}^{2}$ is quadrate of RMS of noise signal $|r(t)|$ that will cause random accesses on RACH surviving the synchronization sequence check and let us consider $K$ as the threshold number. As known, the radio signals on physical layer are modulated using QPSK and GMSK in GSM. It means that two different phases $\theta_{i}$ for $i$ changing from 1 to 2 can be considered and each one codes 1 bit. Therefore to transmit the synchronization sequence containing $K$ bits means to transmit the $k=K / 1$ phase samples (see Fig. 3).

Considering uniform density function for this phase, the probability of sending the phase $\theta_{i}(i \in\langle 1,2\rangle)$ will be $1 / 2$ and probability of sending $k$ samples one after another with the given pattern will be $1 / 2^{k}$.

For the outage probability on $\mathrm{RACH}$ we can write that

$$
p(\rho>Z)=p(A \cap B),
$$

where $A$ is the event that random accesses will survive the synchronization sequence decoding and $B$ is the event that the absolute value of the noise signal with the quadrate of RMS $\sigma_{\text {Total }}^{2}$ exceeds the given threshold $Z$.

Considering that the events $A$ and $B$ are mutually independent means that

$$
p(\rho>Z)=p(A) p(B) .
$$

To calculate the probability of the event $B,(7)$ from the previous chapter can be applied in such a way that the $\sigma^{2}$ will be substituted by $\sigma_{\text {Total }}^{2}$. It means that

$$
p(B)=e^{-\left(Z^{2} / 2 \sigma_{\text {Total }}^{2}\right)} .
$$

Because the probability of the event $A$ is $1 / 2^{k}$, using (11) and (12), (10) leads to the following form

$$
p(\rho>Z)=\frac{1}{2^{k}} e^{-\left(Z^{2} / 2 \sigma_{1 \text { Total }}^{2}\right)} .
$$

The formula (13) can be generalized for the arbitrary PSK modulation in such a way that instead of the constant 2 the number $q$ of different phases enabled using the given PSK will be used and $k=\log _{2}(q)$. For example considering 8 PSK, $k=\log _{2}(8)=3$ as it is demonstrated in Fig. 3.

\section{DISCUSSION AND APPLICABILITY OF THE PROPOSED MODEL PRACTICALLY}

With the proposed mathematical model apart from what mentioned in the previous chapter the following parameters will also be possible to calculate:

- Calculation of the threshold $R A C H B T$ guarantying in advance determined outage probability on RACH using the formula

$$
R A C H B T=10 \log \left(\frac{-2 \ln \left(2^{k} p\left(\rho>P_{T H}\right)\right) \sigma_{\text {Total }}^{2}}{1 m W}\right)(\mathrm{dBm})
$$

and corresponding $P_{T H}$ using the formula

$$
P_{T H}=-2 \ln \left(2^{k} p\left(\rho>P_{T H}\right)\right) \sigma_{\text {Total }}^{2}(\mathrm{~W}) .
$$

The ability to predict the parameter $R A C H B T$ and consequent $P_{T H}$ under condition that the outage probability and $\sigma_{\text {Total }}^{2}$ are known may be very useful in practice. Let us consider a new method implemented within the BTS according to Fig. 4. As it can be seen in the figure the new method consists of four main stages.

- First one is related to $\sigma_{\text {Total }}^{2}$ calculation. Currently the BTS receiver calculates the received power for every RACH burst. The signal power is computed from the received $I$ and $Q$ samples using the formula $p=\sigma_{\text {Total }}^{2}=$ $\frac{1}{N} \sum_{n=0}^{N-1}\left(I_{n}^{2}+Q_{n}^{2}\right)(\mathrm{W})$, where $N$ represents the number of samples involved into the calculation. In principle the formula can be reused for this stage of the method but in order to observe long term behavior the calculation is not

\footnotetext{
${ }^{3}$ This corresponds to the second stage of two procedures method for detecting phantom RACHs as described in the chapter 1 . It should be noted that SNR is measured indirectly by BER of the synchronization suence. For instance if the number of matching bits within the sequence is equal to 7 , then $\mathrm{BER}=7 / 41=17 \%$
} 


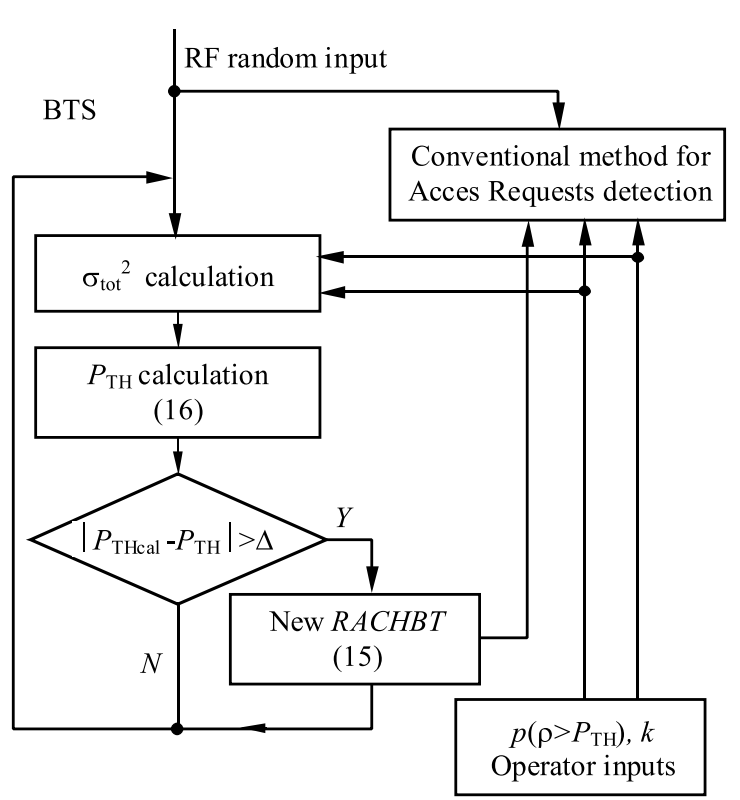

Fig. 4. Principle of a method for $R A C H B T$ prediction for the guaranteed outage probability on $\mathrm{RACH}$

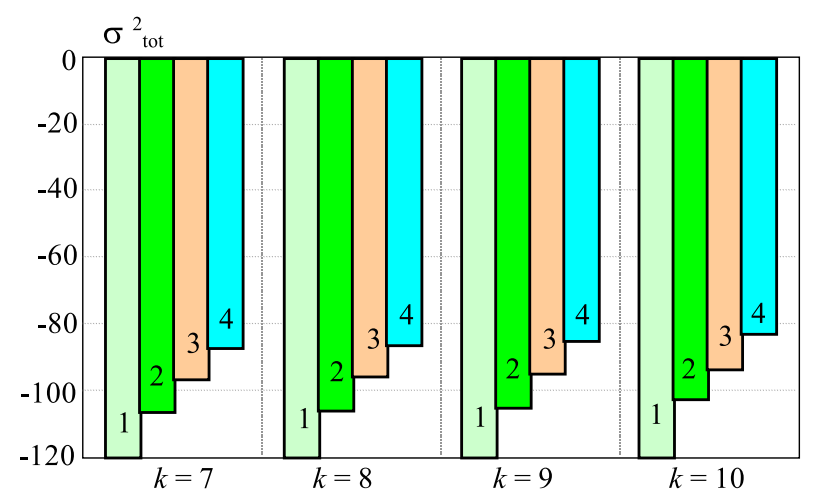

$k=7 \quad k=8 \quad k=9 \quad k=10$

1 RACHBT $=-110-118.66-117.75-116.59-115.02$

$2 R A C H B T=-100-108.66-107.75-106.59-105.02$

$3 \mathrm{RACHBT}=-90 \quad-98.66 \quad-97.75 \quad-96.59 \quad-95.02$

4 RACHBT $=-80 \quad-88.66 \quad-87.75 \quad-86.59 \quad-85.02$

Fig. 5. The $\sigma_{\text {tot }}^{2}$ in $\mathrm{dBm}$ as a function of the RACHBT and $k$

Table 1. Dependence of the RACHBT on the $\sigma_{\text {Total }}^{2}$

\begin{tabular}{cccc}
\hline$p\left(\rho>P_{T H}\right)(\%)$ & $k$ & $\sigma_{\text {Total }}^{2}(\mathrm{dBm})$ & $R A C H B T(\mathrm{dBm})$ \\
\hline 0.02 & 7 & -118.6 & -110 \\
0.02 & 7 & -108.6 & -100 \\
0.02 & 7 & -98.6 & -90 \\
0.02 & 7 & -88.6 & -80 \\
\hline
\end{tabular}

only done from one but from hundreds of RACH bursts. So for the number of samples $N^{*}$ in this case it must hold that $N^{*} \gg N$. The parameter $N^{*}$ is considered to be hard coded.

- The second stage comprises the $P_{T H}$ calculation using
(16). In order to calculate the parameter the $p\left(\rho>P_{T H}\right)$ and $k$ must be known. Regarding the $p\left(\rho>P_{T H}\right)$ we can referee to 3GPP TS 45.005 [5], where is mentioned that for a BTS on a RACH or PRACH with a random $\mathrm{RF}$ input the overall reception performance shall be such that less than $0.02 \%$ of frames are assessed to be error free. ie $p\left(\rho>P_{T H}\right)$ can be set to $2 \times 10^{-4}$. As to parameter $k$ referring to [5] the theoretical BER limits of GMSK and BPSK based on $E_{b} / N_{0}=0 \mathrm{~dB}$, the BER of GMSK is 0.16 . For the GSM where 41 represent the total number of bits in a RACH synchronization sequence this corresponds to ratio $7 / 41$. So the threshold $k$ can be set to 7 . However both $p\left(\rho>P_{T H}\right)$ and $k$ are not considered as hard coded and therefore can be modified by operator. - In the third stage a difference between the calculated power threshold $P_{T H C a l}$ in the previous stage and latest valid $P_{T H}$ is done. If in the absolute value the obtained difference is higher than a $\Delta$ (non zero difference must be used in order to avoid the RACHBT is being changed very often) then from the $P_{T H C a l}$ a new RACHBT is calculated using (15) which is used in the conventional method for the RACH burst detection.

In Table 1 a dependence of the $R A C H B T$ on the $\sigma_{\text {Total }}^{2}$ is shown as an example. As it can be seen for outage probability equal to $0.02 \%$ and $k$ equal to 7 the difference is $8.6 \mathrm{dBm}$.

In Fig. 5 a graph where $\sigma_{\text {Total }}^{2}$ in $\mathrm{dBm}$ as function of the RACHBT and $k$ is shown. The outage probability was for all the scenarios equal to $0.02 \%$. From the obtained results a slight increase of the $\sigma_{\text {Total }}^{2}$ in parallel with the number of matching bits $k$ increase is visible.

\section{CONCLUSION}

A new mathematical model for the evaluation of the invalid access requests probability in mobile communication systems with the focus on GSM has been derived and discussed. Ability to predict a power RACH Busy Threshold (RACHBT) is the most important outcome in relation to keep the probability of the invalid access requests at the desired value. However the mathematical model has a restriction which is that number of components which contribute for resultant noise must be large enough to consider the probability density of the absolute value of the noise is described by the Rayleigh function. It should also be noted that the validity of the proposed method may be affected by the configuration of the network (eg the number of cells using the same Broadcasting Control Channel (BCCH) which are close to the observed cell). Those topics are the good ones for the next research besides the practical implementation of the method and this paper shall be understood as a pioneer work done in this area. 


\section{REFERENCES}

[1] PINES, H. S.-SAYERS, I.-XU, X.-HUANG, W.: Method and system for reducing false detections of access signals, United States Cisco Technology, Inc., US Patent Application 6901116, 2005.

[2] MOUNA-KINGUE, M. S.-SUNDARALINGAM, S.-HASAN, K.-JONES, E.-SAILY, M.-NATH, S. : Detection of invalid channel, US Patent Application 20070010247, 2007.

[3] FELLER, W.: The Fundamental Limit Theorems in Probability, Bull. Amer. Math. Soc. 51 (1945), 800832.

[4] PAPOULIS, A. : Probability, Random Variables, and Stochastic Processes, 2nd ed., McGraw-Hill, New York, 1984, pages 104 and 148.

[5] 3GPP TS 45.005.

Received 23 June 2011

Martin Kollár was born in Spišská Nová Ves, Slovakia, on 7th December 1974. He gained the Ing (MSc) degree in electronics and multimedia telecommunications from the Faculty of Electrical Engineering and Informatics (FEI), Technical University (TU) Košice and a PhD degree in measuring techniques from the FEI TU Košice, in 2000 and 2003, respectively. From 2003 till 2006, he was an assistant professor at the Department of Theory of Electrical Engineering and Measurement, FEI, TU Košice. Since 2006 he has been a senior analyst/architect at Siemens Program and System Engineering and working as an external consultant for Nokia Siemens Networks on the O\&M Performance Measurements area for GSM/GPRS/EDGE and LTE. His research interests include performance measurement techniques for GSM/GPRS/EDGE and LTE, nonlinear circuit theory, smart sensors based on a flip-flop circuit and testing the analog-to-digital converters.

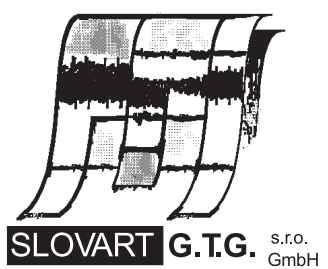

EXPORT - IMPORT
EXPORT - I M P OR T

of periodicals and of non-periodically printed matters, books and CD-ROMs

Krupinská 4 PO BOX 152, 85299 Bratislava 5, Slovakia tel: ++421 263839 472-3, fax: ++421 263839485 info@slovart-gtg.sk; http://www.slovart-gtg.sk

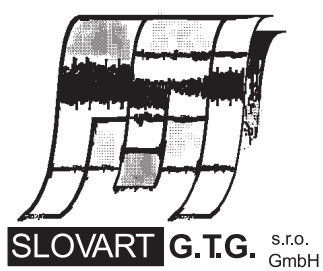

EXPORT - IMPORT 